$\mathrm{RM}-76-4$

PREFERENCE MODELS OF ENVIRONMENTAL IMPACT

Ralph L. Keeney

January 1976

Research Memoranda are interim reports on research being conducted by the International Institute for Applied Systems Analysis, and as such receive only limited scientific review. Views or opinions contained herein do not necessarily represent those of the Institute or of the National Member Organizations supporting the Institute. 


\title{
PREFERENCE MODELS OF ENVIRONMENTAL IMPACT **
}

\author{
Ralph I. Keeney
}

\section{Abstract}

Environmental problems usually involve multiple conflicting objectives, large uncertainties concerning the possible environmental impact, and several individuals or groups whose preferences are very different, but yet very important in choosing an alternative. If one wishes to influence the decision making process using analysis, the above issues should be addressed. One critical aspect, which is usually conducted informally, involves weighing the advantages and disadvantages of the possible impacts of the various alternatives by each of the interested parties.

A multiattribute utility model, called a utility function, quantifies the preferences of an interested party in a manner convenient for integration with an impact model, so that formal evaluation of the alternatives is logical and straightforward. Using the utility functions of the various interested individuals and groups, it is possible to examine the different preference structures and their implications to illuminate the conflicts, focus the discussion, generate creative alternatives, and promote constructive compromises.

In this paper, the basic ideas of multiattribute utility are presented and placed in the context of a decision making framework. The manner in which it aids the analysis of environmental issues is discussed. Several problems having environmental components in which multiattribute utility was used are surveyed. These include siting nuclear power facilities, developing fisheries, controlling a forest pest, examining energy policy, and transporting hazardous materials.

Awareness of environmental problems has certainly increased in recent years. Closely linked to this is the necessity and willingness to do something about the problems. But what? In any particular situation, it is very difficult to sort through the proposed options and identify the best,

\footnotetext{
"Presented at the International Congress of scientists on the Human Environment, Kyoto, Japan, Nov. 16-26, 1975.
} 
or even a reasonable one. Help is needed in deciding exactly what alternatives should be chosen with respect to the environment.

Recognizing this need, governments and many public and private institutions have reacted. In the United States, for example, the National Environmental Policy Act of 1969 required, among other things, that environmental impact statements identify, describe, and evaluate all possible significant environmental consequences of major federally funded or approved projects. Hence, there have been many recent attempts to model large complex environmental systems, both to better understand them and to improve decision making. Some of these efforts do lend substantial support for improving the decisions. However, invariably, they seem to address only a part of the problem. Generally, these models attempt to describe what the environmental impact of any policy may be. They do not include the next step, which requires balancing the pros and cons of the possible impacts in order to reach a decision. The latter function is usually left for the political process, which often means that all the information about the possible impacts is processed informally in the minds of those individuals making the decisions. This paper suggests and illustrates an alternative--a formal model of preferences.

In Section 1, we define the class of problems considered. Specifically, the major factors contributing to their complexity are discussed. Section 2 briefly summarizes the main ideas of multiattribute utility theory. Section 3 indicates the manner in 
which multiattribute utility explicitly addresses the complexities of the preference aspects of environmental problems. Examples are given in Section 4. Conclusions are given in the last section.

\section{The Environmental Problem}

It is critically important to make "good" decisions concerning our environment. In principle, these should depend on two considerations:

1. the likelihoods of the various possible consequences resulting from each of the alternative choices, and

2. the relative desirability of these possible consequences.

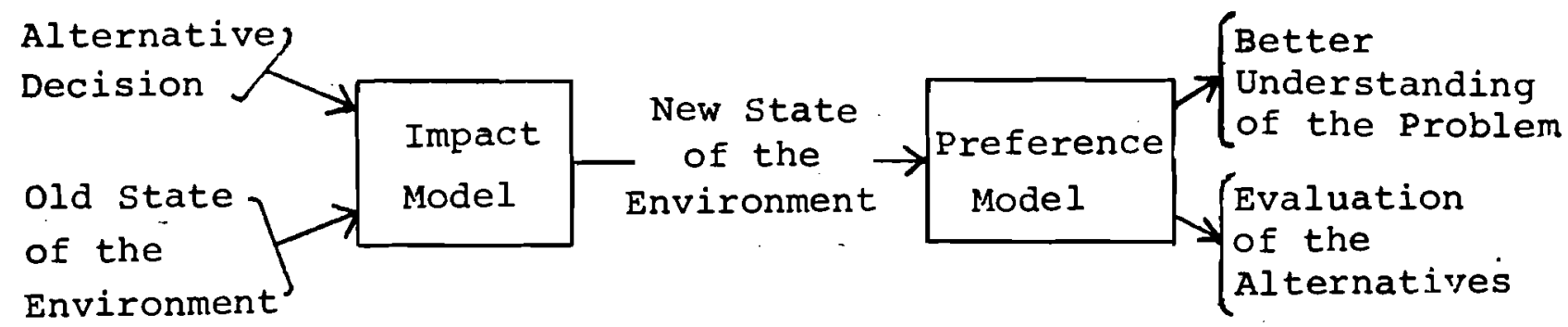

Figure 1. A Composite Model for Analyzing Environmental Problems

Figure 1 conceptualizes these features in a composite model of the decision making process. This composite model comprises two components, an impact model which addresses consideration 1 and a preference model addressing consideration 2 . 
decisions on the state of our environment. For instance, in an air pollution context, models of the implications of each alternative on the sources and amounts of pollution are constructed. These are interrelated with the weather pattern to yield pollution concentrations. Perhaps the implications of the resulting pollution concentrations for human health and ecological systems are included. For each possible alternative, the output of this model would be the consequences indicated in terms of health impact, ecological impact, economic impact, and so on. This information will often be reported probabilistically as it is not possible to predict the environmental impact precisely.

With most environmental models, this is the point where the formal analysis ends. The decision makers are provided with reports and data on the consequences of each alternative and asked to choose among them. It is very difficult to systematically and consistently process all the relevant information in the human mind. The sheer magnitude of most important problems prevents this. For this same reason, namely, the difficulties in logically and consistently thinking through each alternative to arrive at its likely implications, models of environmental impacts are used. With impacts, the idea that a model can be useful in helping to understand the issues and arrive at a "good" decision is often accepted. In this paper, we argue that for the same reasons it can also be very helpful to build a model of preferences.

The preference model is represented by the second box in Figure 1. The inputs to this model are the outputs of the impact model. The outputs of the preference model are intended to be 
a better understanding of the problem as well as an evaluation of the alternatives.

There are a number of complexities which create the need for such a preference model. These include:

- multiple objectives,

- uncertainties about the impact,

- impacts over time,

- many impacted groups with diverse interests,

- multiple decision makers.

Multiple Objectives. In one sense, the environmental problems are problems exactly because of their multiple-objective nature. There is the one objective of society to continually improve its economic and material well-being. In pursuing this objective, the environment is usually detrimentally impacted. The greater the degree to which this occurs, the more aware society becomes of a second major objective: to preserve or improve our natural environment. It is clear that the degree to which we achieve one of these objectives affects the degree to which it is possible to achieve the other. Value tradeoffs indicating how much of one achievement we are willing to forego to attain a fixed amount of another are an important issue.

Uncertainties. At the time decisions are made, it is impossible to precisely forecast the implications of each alternative. often these uncertainties are large. For instance, in evaluating sites for nuclear power facilities, the use of cooling water and the release of heated water back into the river may 
impact the aquatic fauna and flora. These disruptions to local ecological communities may work their way through the ecological system causing detrimental impact in several places. Estimations of the possibilities and magnitudes of such impacts are very difficult. The implication is that the major uncertainties should be formally addressed in a preference model attempting to aid decision makers.

Impacts Over Time. An alternative chosen now may have an impact for many years into the future. This is obvious when consid-ering the interrelationships of various components of the environment. Simply building a dam at a particular site preempts the use of the land for other purposes. A policy of using nuclear power results in radioactive waste, which will remain with us for centuries. Such aspects of the problem are important to address. Preferential concepts such as discounting, the value of time, and intergenerational tradeoffs are involved with this complexity.

Many Impacted Groups. Different individuals have very different preferences. One group of people may be completely willing to sacrifice all the fish in a stream for an additional 1000 megawatt power plant, whereas another group would not be willing to give up one percent of the fish. Those people living near a large power plant are clearly impacted much differently than metropolitan city residents using the generated power miles away from the site. It is important to account for the diverse opinions and priorities that obviously exist in such problems. In some sense, we want to be fair; this implies that tradeoffs 
among the groups must be made.

Multiple Decision Makers. Closely related to the preceding issue is the fact that it is not one decision maker or decision making entity that has the power to select the best decision concerning environmental options. Several parties, often with opposing viewpoints, must collectively arrive at a decision. It is important to provide mechanisms to assist the interaction of the involved decision makers. Perhaps this can help identify and resolve conflicts and lead to "better" decisions for all.

Reviewing this list of complexities, one realizes exactly why it is so difficult to consider all the preferential issues in an environmental problem informally in one's head. Most environmental problems involve aspects of each of the above complexities.

\section{Multiattribute Utility Theory}

The model quantifying one's preferences is called a utility function. When multiple objectives are involved, a measure of effectiveness, or attribute, is needed to indicate the degree to which each objective is met. Hence we have the terminology, multiattribute utility function. This multiattribute utility function is nothing more than an objective function (to be maximized) with one special property: it is scaled in a manner so that in cases involving uncertainty, the expected utility calculated for an alternative is an appropriate measure of the desirability of that alternative. Thus, if one accepts a set of reasonable axioms postulated by von Neumann and Morgenstern [24], 
one should choose the alternative leading to the highest expected utility

The Terminology of Multiattribute Utility. The outputs of the impact model of Figure 1 indicate the degree to which the several objectives might be met in each time period under consideration. To simplify introduction of the ideas, let us focus on one time period and let the set of attributes, which measure the achievement of objectives, be designated by $x_{1}, x_{2}, \ldots, x_{n}$. Then we can let $x_{i}$ be a specific amount of $x_{i}$. For instance, the $i^{\text {th }}$ objective may be to minimize the detrimental impact on fish. An attribute $x_{i}$ to measure this may be the number of $f i s h$ killed, and $x_{i}$ could then be $5000 \mathrm{fish}$. With this notation, the consequence of any alternative is $x \equiv\left(x_{1}, x_{2}, \ldots, x_{n}\right)$. For each alternative $A_{j}$, the impact model gives us a probability distribution $\mathrm{p}_{j}(\mathrm{x})$ indicating which consequences may occur and their likelihoods. This is combined with the utility function $u(\underline{x})$ to calculate the expected utility of alternative $A_{j}$.

The main results of multiattribute utility theory $[6,7,12$, $19,22,23]$ concern representation theorems stating conditions under which a utility function can be expressed in a specific simple functional form. If such a form is appropriate for an analysis, it is then generally much easier to proceed with the assessments necessary to specify the utility function.

Two basic notions used in deriving representation theorems are the concepts of preferential independence and utility independence. Let us define these concepts and then state two representation theorems which have proven to be useful in 
analyzing environmental problems.

Preferential Independence. The pair of attributes $\left\{x_{1}, x_{2}\right\}$ is preferentially independent of the other attributes $\left\{x_{3}, \ldots, x_{n}\right\}$ if preferences among $\left\{x_{1}, x_{2}\right\}$ pairs, given that $x_{3}, \ldots, x_{n}$ are held fixed, do not depend on the level where those attributes are fixed.

Preferential independence implies that the tradeoffs between attributes $x_{1}$ and $x_{2}$ do not depend on $x_{3}, \ldots, x_{n}$.

Utility Independence. The attribute $x_{1}$ is utility independent of the other attributes $\left\{x_{2}, \ldots, x_{n}\right\}$ if preferences among lotteries* over $x_{1}$ (i.e. lotteries with uncertainty about the level of $x_{1}$ only), given that $x_{2}, \ldots, x_{n}$ are held fixed, do not depend on the level where those attributes are fixed.

A main result can now be stated.

Theorem 1. For $n \geq 3$, if for some $x_{i},\left\{x_{i}, x_{j}\right\}$ is preferentially independent of the other attributes for all $j \neq i$ and $x_{i}$ is utility independent of all the other attributes, then either

$$
u(\underline{x})=\sum_{i=1}^{n} k_{i} u_{i}\left(x_{i}\right), \text { if } \Sigma k_{i}=1 \text {, }
$$

or

$$
1+k u(\underline{x})=\prod_{i=1}^{n}\left[1+k k_{i} u_{i}\left(x_{i}\right)\right], \text { if } \sum k_{i} \neq 1
$$

where $u$ and $u_{i}$ are utility functions scaled from zero to one, the $k_{i}$ 's are scaling constants with $0<k_{i}<1$, and $k>-1$ is a nonzero scaling constant.

\footnotetext{
* A lottery is defined by specifying possible consequences which may result and the associated probabilities of their occurrence.
} 
The proof of this result is found in Keeney [12]. Alternative sets of assumptions leading to either form (1) or (2) are found in Fishburn [6], Meyer [19], and Pollak [22]. The functional form (1) is the additive utility function and (2) is the multiplicative utility function. For the case of two attributes, the following is proved in Keeney [11].

Theorem 2. For $n=2$, if $x_{1}$ is utility independent of $x_{2}$ and $\mathrm{x}_{2}$ is utility independent of $\mathrm{x}_{1}$, then the utility function $u\left(x_{1}, x_{2}\right)$ is either additive or multiplicative.

To use either the additive or multiplicative form, we need to obtain exactly the same information, the $\mathrm{n}$ single-attribute utility functions $u_{i}$ and the $n$ scaling constants $k_{i}$. Assessing and using this information is discussed in detail in Keeney and Raiffa [16].

In terms of the required assessments and general robustness, the additive and multiplicative utility functions appear to be the practical ones for say $n \geq 4$. Even when the requisite assumptions do not precisely hold over the domains of all the attributes, it may be a good approximation to assume they do, or it may be reasonable to integrate different additive and multiplicative utility functions over separate regions of these attributes. More general functional forms, requiring more assessments, have been developed using a similar approach for cases requiring additional flexibility in the preference structure; see, for example, Bell [2], Farquhar [5], Fishburn [8], Keeney [11], Kirkwood [18], and Oksman [21]. 


\section{Addressing the Complexities}

It is important to describe briefly how a preference model--a multiattribute utility function--addresses the five complexities outlined in section 1 .

Multiple Objectives. The decision maker's utility function u is assessed such that $u\left(x_{1}, x_{2}, \ldots, x_{n}\right) \geq u\left(x_{1}^{\prime}, x_{2}^{\prime}, \ldots, x_{n}^{\prime}\right)$ if and only if $\left(x_{1}, x_{2}, \ldots, x_{n}\right)$ is preferred to $\left(x_{1}^{\prime}, x_{2}^{\prime}, \ldots, x_{n}^{\prime}\right)$. with $u$ we can easily construct the indifference curves over $\left\{x_{1}, x_{2}, \ldots, x_{n}\right\}$. This indicates exactly how much of one attribute one is willing to give up in order to get a specified amount of another attribute. That is, the value tradeoffs are made explicit.

Uncertainties. As indicated, the utility function is quantified in a special manner so that the expected utility of an alternative is an appropriate indicator of the desirability of that alternative. To use average values of the attributes per se rather than the utility over the attributes is often clearly inappropriate. Suppose, for example, two alternatives were identical except for the fact that one resulted in fifty percent of the fish in a stream dying and the other resulted in an equal chance of either all or none dying. Both options imply an expected loss of fifty percent, but most people would still prefer a sure loss of fifty percent. An appropriate utility function captures such preferences.

Impacts Over Time. Let us now expand an attribute set to be $\left\{x_{i}^{t}: i=1, \ldots, n ; t=1, \ldots, T\right\}$ where $x_{i}^{t}$ is the impact on the $i^{t h}$ attribute in time period $t$. If we now assess the decision maker's utility 
function $u\left(\underline{x}^{1}, \underline{x}^{2}, \ldots, \underline{x}^{T}\right)$, we have explicitly identified all the value tradeoffs among attributes over time. For problems such as radioactive waste, the utility function will indicate whether the 'benefits' in the near future are worth the 'costs' in the distant future. To examine this, one compares the utility of energy programs involving much waste and those involving none.

Many Impacted Groups. An overall measure of the degree to which each group is impacted by any consequence is its utility for that consequence. Thus if utility functions $u_{j}, j=1,2, \ldots, J$, are assessed for the $\mathrm{J}$ groups, the decision maker can assess his utility function $u\left(u_{1}, u_{2}, \ldots, u_{J}\right)$. This formulation explicitly focusses on the value tradeoffs that the decision maker must make among groups. The issue is how much should be taken away from one group to satisfy another group. Concepts of fairness and equity play important roles here.

Multiple Decision Makers. Now suppose that rather than there being an overall decision maker with utility function $u$, the $J$ groups are each participants in the decision making process. By analyzing their utility functions $u_{j}$, one can identify areas of agreement and disagreement. This may in itself suggest creative new options which all groups like. More likely, it will provide a solid base from which to begin constructive compromise.

We must mention the obvious, that utility is not a panacea for 'solving' some very important problems. However, utility theory does address, rather than avoid, some of the complications 
ever present in environmental problems. There is much to be learned: our understanding of the problems needs to be improved; our theory and techniques need to be sharpened; and our interpretation and use of the analysis need to be scrutinized. The examples in the next section briefly describe some initial attempts in this direction.

\section{Applications}

Here, we will briefly summarize studies in five separate problem areas where multiattribute utility analysis has been used. All of the studies addressed the multiple-objective and uncertainty complexities. In addition, most of them addressed one of the other complexities. To date, there is essentially no experience in addressing all the complexities in the same problem.

Nuclear Power siting, Two studies have been conducted of nuclear power plant siting using multiattribute utility. In a doctoral dissertation, Gros [9] studied the problem of deploying 1,00O-Mw base-load units on the New England coast. Specifically, he examined the benefits and costs accruing to four separate groups: environmentalists, the utility companies, regulatory agencies, and local groups. Using individuals with a knowledge of each group's interests, utility functions for each of these were assessed over four proxy attributes: monetary costs, population within fifteen miles of the site, temperature of water released after cooling, and capacity of the site measured in number of $1,000-$ Mw units.

Using the overall approach described in Nair et al. [20], 
Woodward-Clyde Consultants of San Francisco have been using multiattribute decision analysis in their professional practice while consulting for utility companies. The first study was recently completed $[15,25]$. First a series of screening models was used to select candidate sites, considering several factors such as earthquake potential, faults, water availability, etc. Evaluation of these sites involved assessing a utility function including the environmental impact on fish, waterfowl, and rare and endangered species; the socio-economic impact on the communities near the site due to the boom-bust cycle; the safety of the population from radiation and possible accidents; costs; and system reliability.

To illustrate the approach, consider one environmental objective of the study, "to minimize adverse impact on salmonids." Obvious measures such as the number of fish killed were not entirely appropriate, since the loss of $1000 \mathrm{fish}$ in a stream of 1000 is more disastrous than the loss of the same number of fish in a stream of $50,000 \mathrm{fish}$. The percent killed is also important since the genealogy of fish in each stream is slightly different. However, the percent of fish killed, used alone, is not adequate to measure the objective. For streams with less than 100,000 fish, a preference model--a utility function--was constructed including both the percent of fish in the stream killed and the number of fish in the stream. The assumptions necessary for Theorem 2 were verified and the model (2) was calibrated. For streams with more than 100,000 salmonids, it was virtually impossible to eliminate as much as 50 percent of 
the salmonid so the percent was not important. For such streams, a one-attribute utility function for the number of fish killed was used. The two utility models were then consistently scaled. Details of all these assessments are in Keeney and Robilliard [17].

Forest Pest Management. The ecology project at the International Institute for Applied Systems Analysis in Laxenburg, Austria, developed a model for evaluating control strategies for the spruce budworm. This pest periodically destroys much of the forests of New Brunswick, Canada. Major variables of interest impacted by the alternative strategies are the lumber industry profits in New Brunswick, the unemployment in the area (since the lumber industry is very significant), and the recreational value of the forest. The problem is complicated because the impact of any strategy affects periods of several years. Bell $[1,3]$ reports a detailed effort to assess a utility function over these three attributes over time for purposes of evaluating treatment alternatives.

Control of Fisheries. The Skeena River in British Columbia, Canada, is an important salmon fishing area. The Canadian Department of the Environment has the responsibility for policy decisions including who can fish, what salmon species and size they can catch, where they can fish, which methods they can use, and when they can fish. Such decisions impact directly or indirectly everyone living in the skeena area. As part of the initial stages in examining this problem, first-cut utility 
functions of the five main impacted groups were formalized using individuals familiar with their interests. Environmental considerations such as the diversity of salmon in the skeena were examined relative to economic indicators such as the annual income of a fisherman and the cost of salmon per pound.

With two members of the study team, preliminary utility functions addressing the value tradeoffs between groups has been assessed [13]. By examining these two preference models, some important disagreements were identified. These are in the process of being reconciled among team members. Although the context is different, the spirit of identifying and resolving differences in this manner is the same as that suggested for addressing the complexity of multiple decision makers.

Transporting Hazardous Materials. In recent years, there has been a large increase in the volume of hazardous materials being transported within the United States. In an attempt to evaluate which modes of surface transportation--rail, highway, water, and pipeline--were most safe under given conditions, Kalelkar et al. [10] found it necessary to develop a subjective index of possible environmental effects due to hazardous chemical spills. The scale is presented in Table 1 to illustrate the general idea of such a scale. It allows one to combine the impact due to several sources into one measure. For example, this scale includes odors, leaf damage, loss of foliage, and short and long term impacts on animals. It is important to increase our willingness to use and skill in constructing subjective scales. Another example of a subjective scale concerns the biological impacts (except for salmonids) of the nuclear power siting study of Woodward-Clyde Consultants [25]. 
Table 1. Environmental Effects from Hazardous Chemical Spills

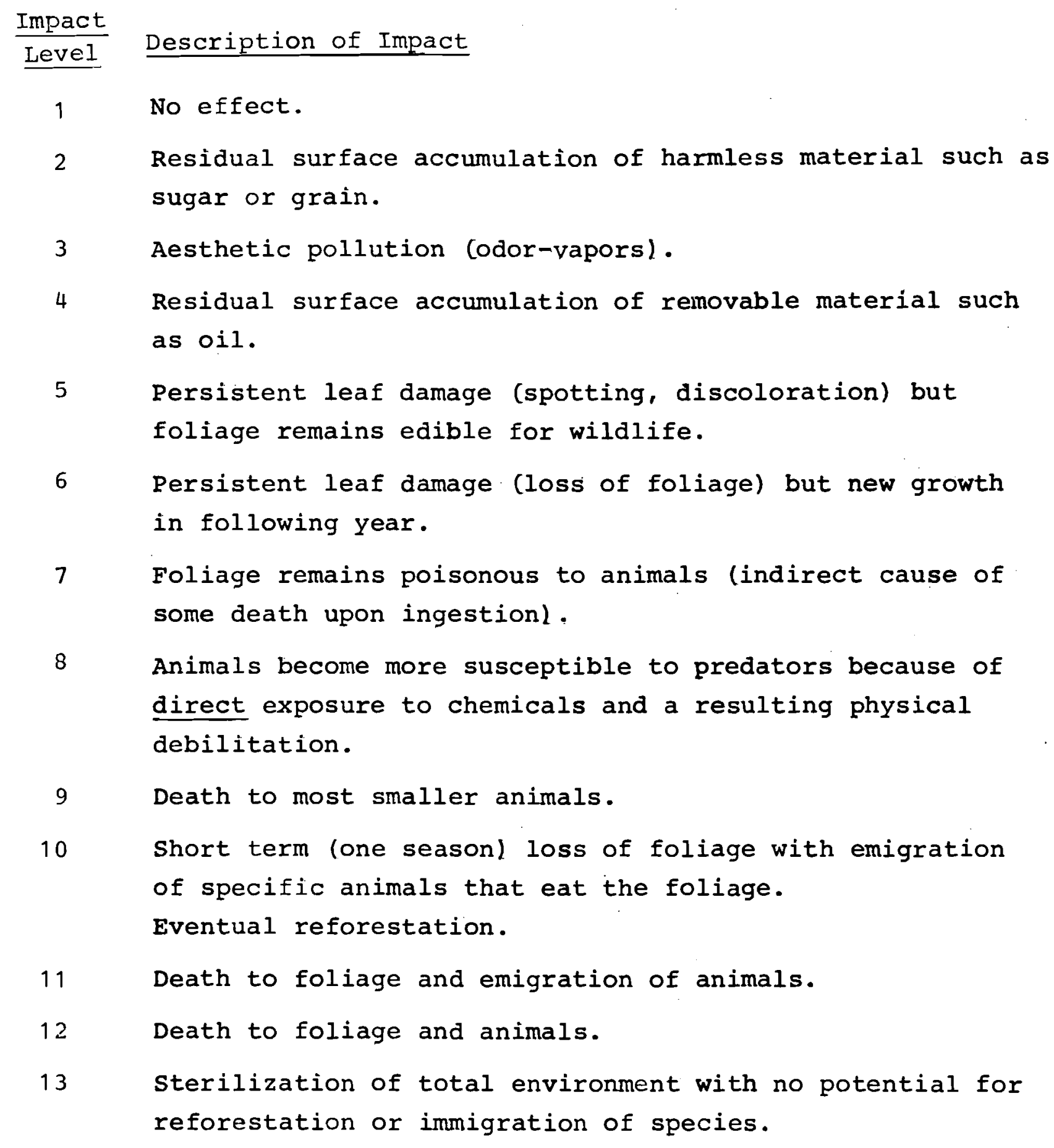


Energy Policy. In making energy policy decisions, value tradeoffs among several environmental variables are critical. The environmental consequences of a policy of introducing nuclear power plants at a rapid rate is much different than one limiting the use of nuclear power in favor of coal plants. Buehring [4] has constructed an environmental impact model indicating the likely effects of various policies in terms of eleven variables including deaths, $\mathrm{SO}_{2}$ pollution, radioactive waste, health effects, water used, land used, and electrical energy generated. A preference model to integrate with the environment impact model was developed. Details of the preference assessments are found in Keeney [14].

\section{Recommendations}

It is a fact that most environmental problems do involve the five complexities outlined in section 1. Hence if one considers analysis worthwhile, the question is whether to include these aspects, formally or informally. Multiattribute utility theory is developed to the degree where it can be of substantial help in understanding and resolving these complexities. The art of implementing the theory is currently being improved.

In many complicated problems, several man-years may be spent developing an impact model (e.g. simulation) to identify the possible consequences of each alternative. The final result of all this is a report to "the decision maker" who, after perhaps a week of thinking and consulting with his "advisors," makes a decision. If the problem requires so much 
modelling, it seems that in some cases it may be very difficult to sort out in a week the overall preferences in one's head. Assessing a multiattribute utility function offers the decision maker an alternative to doing an in-the-head analysis. This helps him to both (1) get his preferences straight, and (2) evaluate policies using his preferences and the impact model output in a logically consistent manner. It would appear that for some problems, the shifting of a few man-months effort from the modelling aspects to the preference aspects would prove to be worthwhile.

The experience with developing preference models indicates that the process itself can be very useful. The process assists in articulating substantive issues, sensitizing different individuals to the issues involved, generating creative alternatives, communicating, isolating differences in judgment and preferences, and resolving these differences. This should itself promote better decision making.

\section{$\underline{\text { References }}$}

1. Bell, D.E. "Defining and Quantifying Goals for Measuring the state of an Ecosystem." RR-74-26, IIASA, Laxenburg, Austria, 1974.

2. Bell, D.E. "A Utility Function for Time streams Having Inter Period Dependencies." RR-75-22, IIASA, Laxenburg, Austria, 1975.

3. Bell, D.E. "A Utility Function Over Time for Forest Pest Management." Research Memorandum, IIASA, Laxenburg, Austria, 1976.

4. Buehring, W.A. "A Model of Environmental Impacts from Electrical Energy Generation in Wisconsin," unpublished Ph.D. dissertation, Department of Nuclear Engineering and Institute of Environmental Studies, University of Wisconsin, Madison, 1975. 
5. Farquhar, P.H. "The Fractional Hypercube Decomposition Theorem for Multiattribute Utility Functions." Operations Research, 23 (1975), pp. 941-967.

6. Fishburn, P.C. "Independence in Utility Theory with Whole Product Sets." Operations Research, 13 (1965), pp. 28-45.

7. Fishburn, P.C. Utility Theory for Decision Making. New York, wiley, 1970 .

8. Fishburn, P.C. "Bernoullian Utilities for Multiple-factor Situations." In Multiple Criteria Decision Making, Eds., J.L. Cochrane and M. Zeleny. University of South Carolina Press, Columbia, South Carolina, 1973.

9. Gros, J.G. "A Paretian Environmental Approach to Power Plant Siting in New England." Harvard University, Cambridge, Massachusetts, 1974.

10. Kalelkar, A.S., Partridge, L.J., and Brooks, R.E. "Decision Analysis in Hazardous Material Transportation," Arthur D. Little, Incorporated, Cambridge, Mass., 1974.

11. Keeney, R.L. "Utility Functions for Multiattributed Consequences." Management Science, 18 (1972), pp. 276-287.

12. Keeney, R.L. "Multiplicative Utility Functions." Operations Research, 22 (1974), pp. 22-34.

13. Keeney, R.L., "A Utility Function for Examining Policy Affecting Salmon in the Skeena River," Research Memorandum, IIASA, Laxenburg, Austria, 1976.

14. Keeney, R.L. "Energy Policy and Value Tradeoffs," Research Memorandum, IIASA, Laxenburg, Austria, 1976.

15. Keeney, R.L., and Nair, K. "Selecting Nuclear Power Sites Using Decision Analysis," Woodward-Clyde Consultants, San Francisco, California, 1975.

16. Keeney, R.L., and Raiffa, H. Decisions with Multiple Objectives. New York, wiley, forthcoming, 1976.

17. Keeney, R.L., and Robilliard, G.A. "Assessing and Evaluating Environmental Impacts at Proposed Nuclear Power Sites," Woodward-Clyde Consultants, San Francisco, California, 1975.

18. Kirkwood, C.W. "Parametrically Dependent Preferences for Multiattributed Consequences." Operations Research, forthcoming January-February 1976. 
19. Meyer, R.F. "On the Relationships Among the Utility of Assets, the Utility of Consumption, and Investment Strategy in an Uncertain, but Time Invariant World." Proceedings of the Fourth IFORS Conference. Venice, Italy, 1969 .

20. Nair, K., Brogan, G.E., Cluff, L.S., Idriss, I.M., Mao, K.T. "An Approach to the Siting of Nuclear Power Plants." In Siting of Nuclear Facilities, International Atomic Energy Agency, Vienna, Austria, 1975.

21. Oksman, W. "Markov Decision Processes with Utility Independent Objective Functions." Dissertation, Harvard University, Cambridge, Massachusetts, 1974.

22. Pollak, R.A. "Additive von Neumann-Morgenstern Utility Functions." Econometrica, 35 (1967), pp. 485-595.

23. Raiffa, H. "Preferences for Multi-Attributed Alternatives." RM-5868-DOT/RC, RAND Corporation, April 1969.

24. von Neumann, J., and Morgenstern, O. Theory of Games and Economic Behavior. Second Edition. Princeton, New Jersey, Princeton University Press, 1947.

25. Woodward-Clyde Consultants,"Final Report to the Washington Public Power Supply System! San Francisco, Cal., 1975. 